\title{
Eficiência do Triclopyr no Controle de Plantas Daninhas em GRAMADO (Paspalum notatum) ${ }^{1}$
}

\author{
Triclopyr Efficiency in Controlling Weeds in the Lawn (Paspalum notatum)
}

\author{
FREITAS, F.C.L. ${ }^{2}$, FERREIRA, L.R. ${ }^{3}$, SILVA, A.A. ${ }^{3}$, BARBOSA, J.G. ${ }^{3}$, MIRANDA, G.V. ${ }^{3}$ e \\ MACHADO, A.F.L. ${ }^{4}$
}

\begin{abstract}
RESUMO - As plantas daninhas interferem nos gramados, prejudicando a sua formação, qualidade e estética. O carrapicho-beiço-de-boi (Desmodium incanum) e a zórnia (Zornia latifolia), quando não controlados, podem atingir níveis de infestação que chegam a dizimar o gramado. Dentre as diversas técnicas de manejo de plantas daninhas em gramados, destaca-se o emprego de herbicidas; todavia, as informações sobre a utilização desses produtos em gramados são escassas. O objetivo deste trabalho foi avaliar a eficiência do herbicida triclopyr no controle de Desmodium incanum e Zornia latifolia, infestantes da grama-batatais (Paspalum notatum). O trabalho foi desenvolvido em gramado estabelecido no campus da Universidade Federal de Viçosa, em Viçosa-MG, entre os meses de abril e maio de 2000. Os tratamentos avaliados foram seis doses do triclopyr $(0,00 ; 0,24 ; 0,48 ; 0,72 ; 0,96$; e $1,20 \mathrm{~kg}$ hả) e uma dose do 2,4$\mathrm{D}+$ picloram $(0,48+0,128 \mathrm{~kg} \mathrm{ha})$, dispostos em delineamento experimental com blocos ao acaso e quatro repetições. O tamanho de cada parcela foi de $12 \mathrm{~m}^{2}(4,0 \times 3,0 \mathrm{~m})$, sendo os tratamentos aplicados em uma faixa de $2,0 \mathrm{~m}$ de largura, ficando $1,0 \mathrm{~m}$ como testemunha lateral, para auxiliar nas avaliações visuais. Foram realizadas avaliações de fitotoxicidade aos 10 e 20 DAT (Dias Após o Tratamento) e nível de controle aos 15 e 30 DAT, ambos com base na testemunha lateral. Em nenhum dos tratamentos avaliados verificaram-se danos na qualidade do gramado. A partir das doses de 0,48 e $0,66 \mathrm{~kg}$ hal de triclopyr foram obtidos controles iguais ou superiores a $90 \%$ de Desmodium incanum e Zornia latifolia, respectivamente. Esses resultados foram semelhantes ao do tratamento-padrão $(2,4$-D + picloram a 0,48 + 0,128 $\mathrm{kg} \mathrm{ha} \mathrm{h}^{1}$. Concluiu-se que o triclopyr mostrou-se seletivo para grama-batatais, proporcionando excelente controle das plantas daninhas acima mencionadas.
\end{abstract}

Palavras-chave: Desmodium incanum, Zornia latifolia, Paspalum notatum, 2,4-D, picloram.

\begin{abstract}
Weeds interfere in the formation, quality and aesthetics of the lawn. Desmodium incanum, and Zornia latifolia, when left uncontrolled, can reach infestation levels that would kill the lawn. Herbicide application is one of the most effective ways of handling weeds in lawn; however, information on the use of these products in lawns is scarce. The objective of this work was to evaluate the efficiency of the herbicide triclopyr in controlling Desmodium incanum and Zornia latifolia in the lawn Bahiagrass (Paspalum notatum). The experiment was developed in a lawn area of the campus of the Federal University of Viçosa, in Viçosa-MG, Brazil, from April to May 2000. The treatments were six doses of triclopyr $10.00 ; 0.24 ; 0.48 ; 0.72 ; 0.96$ and $\left.1.20 \mathrm{~kg} \mathrm{ha}^{-1}\right)$ and one dose of the 2,4-D + picloram $\left(0.48+0.128 \mathrm{~kg} \mathrm{ha}^{-1}\right)$, arranged in a randomized complete block design, with four repetitions. The size of each plot was $12 \mathrm{~m}^{2}(4.0 \times 3.0 \mathrm{~m})$, with the treatments applied in a $2.0 \mathrm{~m}$ wide strip, with $1.0 \mathrm{~m}$ as lateral check to aid in the visual evaluations. Phytotoxicity evaluations were accomplished at 10 and 20 DAT (days after treatment) and the control level at 15 and 30 DAT, both based on the lateral chek. None of the treatments studied showed damage to the quality of the lawn. Starting with doses of 0.48 and 0.66 triclopyr $\mathrm{kg} \mathrm{ha}^{-1}$, controls similar or superior to $90 \%$ of Desmodium incanum and Zornia latifolia were obtained, respectively. These results were similar to that of the standard treatment $(2,4-D+$ picloram for $\left.0.48+0.128 \mathrm{~kg} \mathrm{ha}^{-1}\right)$. It was concluded that triclopyr is a selective herbicide for Bahiagrass, providing excellent control of the weeds studied.
\end{abstract}

Key words: Desmodium incanum, Zornia latifolia, Paspalum notatum, 2,4-D, picloram.

Recebido para publicação em 29.4.2002 e na forma revisada em 2.4.2003.

2 Doutorando do Dep. de Fitotecnia da Universidade Federal de Viçosa - UFV, Bolsista do CNPq - Brasil, 36571-000 Viçosa-MG; ${ }^{3}$ Profs. do Dep. de Fitotecnia da UFV; ${ }^{4}$ Estudante de Agronomia da UFV. 


\section{INTRODUÇÃO}

As plantas daninhas interferem de várias formas nos gramados: prejudicam a sua formação, condução e estética e concorrem por água, luz, nutrientes e espaço físico, chegando em muitos casos a dizimá-los por completo. A introdução das plantas daninhas ocorre, na maioria das vezes, no ato da implantação do gramado, principalmente naqueles constituídos pela grama-batatais (Paspalum notatum), espécie predominante na região centro-sul do Brasil (Demattê, 1983), cuja propagação é feita de forma assexuada por meio de mudas normalmente originárias de áreas de pastagens, comumente infestadas por diversas espécies de plantas daninhas. Também, a infestação pode ser favorecida pela degradação do gramado, em função da compactação do solo e de cortes sucessivos, com a retirada da grama aparada sem a devida reposição dos nutrientes.

A grama-batatais é uma planta com mecanismo fotossintético do tipo $\mathrm{C}_{4}$, que requer altas taxas de luminosidade e temperaturas elevadas para o seu pleno desenvolvimento. Em épocas do ano com temperaturas mais amenas e menores taxas de luminosidade, tem o desenvolvimento reduzido, o que acarreta maior competição das plantas do tipo $\mathrm{C}_{3}$, que se desenvolvem bem nessas condições, necessitando de intervenção no sentido de reduzir a interferência. Dentre as plantas daninhas infestantes destacam-se o carrapicho-beiço-deboi (Desmodium incanum) e a zórnia (Zornia latifolia), que, de acordo com Kissmann (1997) e Lorenzi \& Souza (2000), têm sido freqüentes em gramados formados pela grama-batatais.

De acordo com Lorenzi \& Souza (2000), a espécie Desmodium incanum possui os seguintes nomes comuns: carrapicho-beiço-de-boi, pega-pega, amores-do-campo, baba-de-boi, marmelada-de-cavalo, amores-de-vaqueiro, carrapicho, agarra-agarra e mata-pasto. É uma planta perene, herbácea, prostrada ou ascendente, de caule pubescente, com 30-60 cm de comprimento e vagem aderente de $2 \mathrm{a} 3 \mathrm{~cm}$ de comprimento, que se propaga por sementes. É nativa da América tropical e ocorre em quase todo o Brasil, sendo encontrada em gramados, beira de estradas e culturas perenes, presente freqüente em gramados e pastagens formados pela espécie Paspalum notatum.

Planta Daninha, Viçosa-MG, v.21, n.1, p.159-164, 2003
A Zornia latifolia é uma angiospermae da família leguminosae-papilionoideae popularmente conhecida como urinária, erva-deovelha, alfafa-do-campo, carrapicho, chapinha, ubiruana, urinana e zórnia. É uma planta perene, herbácea, prostrada, ramificada, de ramos asparso-seríceos, de 30 a $60 \mathrm{~cm}$ de comprimento e se propaga apenas por sementes. É nativa do Brasil e sua ocorrência é muito freqüente em todas as regióes tropicais brasileiras, podendo ser encontrada em gramados mal manejados, beira de carreadores e terrenos baldios (Lorenzi \& Souza, 2000).

Dentre os métodos de controle de plantas daninhas, o mecânico por meio de roçadas e arranque manual é o mais utilizado em gramados. Entretanto, espécies como Desmodium incanum e Zornia latifolia adaptadas a cortes baixos com alta capacidade de rebrota requerem roçadas freqüentes. $O$ arranque em áreas extensas, com alto nível de infestação, é inviável, por apresentar custo elevado e pela dificuldade operacional. O controle químico por meio de herbicidas é uma alternativa que facilita a manutenção dos gramados, melhorando o seu aspecto e reduzindo os custos.

Segundo Christoffoleti \& Aranda (2001), o controle de plantas daninhas por meio de herbicidas depende da seleção de produtos eficientes no controle, porém sem causar injúria ao gramado. No entanto, pesquisas no Brasil sobre a seletividade de herbicidas para gramados são ainda muito escassas.

Dentre os produtos com elevado potencial de uso para gramados destaca-se o triclopyr (Mc Cartye et al., 1996; Mislevy, et al., 1997). O éster butoxietílico do ácido 3,5,6-tricloro-2piridiloxiacético (triclopyr) é um herbicida sistêmico com absorção foliar e radicular. É utilizado para o controle de plantas daninhas latifoliadas em pastagens e na cultura do arroz. Sua degradação no solo é rápida, com meiavida de 20 a 45 dias, dependendo do tipo de solo e das condições climáticas. Em solos com pH mais baixo o seu grau de adsorção é mais elevado e a mobilidade é menor. Sua degradação ocorre mais rapidamente em condições de umidade e temperatura mais elevadas (Rodrigues \& Almeida, 1998).

O sal trietanolamina do ácido 4-amino 3,5,6 tricloropicolínico (picloram) é um produto 
extremamente ativo sobre dicotiledôneas, sendo utilizado em misturas com o 2,4-D para controlar plantas daninhas anuais, perenes, arbustos e árvores em pastagens. É fracamente adsorvido pela argila e pela matéria orgânica. Apresenta longa persistência e fácil mobilidade no solo, podendo se acumular no lençol freático raso, em solos de textura arenosa (Silva et al., 2001). Sua meiavida no solo é de 90 dias, variando de 20 a 300 dias (Rodrigues \& Almeida, 1998).

De acordo Mislevy et al. (1997), as doses de triclopyr utilizadas no controle de plantas daninhas em gramados têm variado de 0,56 a $1,12 \mathrm{~kg} \mathrm{ha}{ }^{1}$. A aplicação seqüencial de 0,56 + $0,56 \mathrm{~kg} \mathrm{ha}^{-1}$ proporcionou, segundo este autor, excelente controle de Solanum viarum sem efeito fitotóxico ao gramado aos 30 dias após a aplicação, com resultados similares aos da dose de $1,12 \mathrm{~kg} \mathrm{ha}^{1}$ aplicada de uma única vez. O mesmo autor, em 1999, obteve $100 \%$ de controle de Solanum viarum na dose única de $0,60 \mathrm{~kg}$ hà de triclopyr. Também, Mullahey et al. (1993) verificaram que o triclopyr a $1,12 \mathrm{~kg}$ hà controlou $99 \%$ do Solanum viarum em gramados com as espécies Paspalum notatum e Digitaria decumbens pelo período de 90 dias após o tratamento, sem prejudicar a qualidade. Resultados excelentes com este herbicida foram também observados por Mc Cartye et al. (1996), os quais verificaram controle satisfatório para a espécie Robus argutus em grama-batatais com triclopyr a $0,56 \mathrm{~kg} \mathrm{ha}^{1}$ pelo período de 52 semanas após o tratamento, com mínimos danos ao gramado.

Avaliando a eficiência de diversos herbicidas no controle do Solanum viarum em grama-batatais, Mullahey et al. (1993) e Akanda et al. (1997) consideraram satisfatório o índice de controle superior a $90 \%$.

Este trabalho teve como objetivo avaliar a seletividade do herbicida triclopyr para grama-batatais e sua eficácia no controle de Desmodium incanum e Zornia latifolia.

\section{MATERIAL E MÉTODOS}

O trabalho foi desenvolvido no campus da Universidade Federal de Viçosa, em Viçosa-MG, no período de abril a maio de 2000 , em gramado estabelecido com a grama-batatais (Paspalum notatum), infestado por Desmodium incanum e

\section{Zornia latifolia.}

O delineamento experimental foi de blocos inteiramente casualizados, com quatro repetições e sete tratamentos, sendo seis doses do triclopyr $(0,00 ; 0,24 ; 0,48 ; 072 ; 0,96$ e $1,20 \mathrm{~kg}$ hả) e um tratamento-padrão com o 2,4-D + picloram $\left(0,48+0,128 \mathrm{~kg} \mathrm{ha}^{1}\right)$. Os produtos comerciais utilizados foram o Garlon ( $480 \mathrm{~g} \mathrm{~L}^{-1}$ de triclopyr) e o Tordon ( $240 \mathrm{~g} \mathrm{~L}^{-1} \mathrm{de}$ 2,4-D + $64 \mathrm{~g} \mathrm{~L} \mathrm{~L}^{-1}$ de picloram).

A área total de cada parcela foi de $12,0 \mathrm{~m}^{2}$, com 4,0 m de comprimento por 3,0 m de largura. Os tratamentos foram aplicados em uma faixa de $2,0 \mathrm{~m}$ de largura, ficando a faixa de $1,0 \mathrm{~m}$ como testemunha lateral, para auxiliar nas avaliações visuais. A bordadura da área aplicada da parcela foi de $0,50 \mathrm{~m}$, resultando em 3,0 m² de área útil.

Os tratamentos foram aplicados por meio de pulverizador costal, munido com uma barra de quatro bicos de jato plano ("leque") 11002, espaçados entre si de $0,5 \mathrm{~m}$, à pressão constante (mantida pelo $\mathrm{CO}_{2}$ comprimido) de $2,1 \mathrm{kgf} \mathrm{cm}^{2}$, proporcionando um volume de calda de $200 \mathrm{~L}$ ha'. Durante a aplicação, as parcelas foram protegidas lateralmente com uma lona plástica, para se evitar deriva da calda pulverizada para as parcelas laterais.

A toxicidade no gramado foi determinada aos 10 e 20 dias após o tratamento (DAT), por avaliação visual, utilizando-se a escala de 0 a 100 , sendo " 0 " ausência de fitotoxicidade e "100" morte das plantas.

Os níveis de controle das plantas infestantes para Desmodium incanum e Zornia latifolia foram verificados aos 15 e 30 DAT, por meio de avaliação visual em relação à testemunha lateral, utilizando-se a escala de 0 a 100 , sendo "0" ausência de controle e "100" controle total das plantas daninhas.

Os dados referentes ao nível de controle foram submetidos às análises de variância e as doses do triclopyr foram também avaliadas por meio de análise de regressão, em que foram testados os efeitos linear e quadrático da raiz quadrada do modelo polinomial, ou seja, $\mathrm{y}=\mathrm{a}+\mathrm{bx}^{0,5}+\mathrm{cx}$, pelo teste $\mathrm{F}$ a $5 \%$ de probabilidade. O modelo adotado foi devido à explicação biológica, à significância do quadrado médio da regressão e aos coeficientes de regressão.

Os dados referentes à avaliação visual de

Planta Daninha, Viçosa-MG, v.21, n.1, p.159-164, 2003 
toxidez no gramado estão apresentados na forma de tabelas, não sendo analisados estatisticamente.

\section{RESULTADOS E DISCUSSÃO}

Os resultados das avaliações de toxidez dos tratamentos aos 10 e 20 DAT na grama-batatais estão apresentados na Tabela 1. Observase que o triclopyr nas doses de até $0,72 \mathrm{~kg} \mathrm{ha}^{1}$ não apresentou sintomas visuais nas épocas avaliadas; entretanto, para os tratamentos com o triclopyr nas doses de 0,96 e $1,20 \mathrm{~kg} \mathrm{ha}^{1} \mathrm{e}$ para o $2,4-\mathrm{D}+$ picloram na dose de $480+$ $128 \mathrm{~g}$ ha, verificou-se, aos 10 DAT, toxicidade de até $10 \%$, considerada leve. Aos 20 DAT não foi verificado efeito fitotóxico dos tratamentos estudados. Estes resultados estão de acordo com os encontrados por Mullahey et al. (1993) e Mislevy et al. (1997), que não verificaram nenhum efeito fitotóxico no gramado quando se aplicou triclopyr na dose de $1,12 \mathrm{~kg}$ had.

O tratamento-padrão (2,4-D + picloram a $0,48+0,128 \mathrm{~kg} \mathrm{ha}^{1}$ ) proporcionou controles de $88 \%$ aos 15 DAT e $93 \%$ aos 30 DAT para Desmodium incanum e de $84 \%$ aos 15 DAT e $90 \%$ aos 30 DAT para Zornia latifolia, semelhante aos melhores controles obtidos pelo triclopyr. Esses resultados, com níveis de controle iguais ou superiores a $90 \%$ aos 30 DAT, podem ser considerados satisfatórios, conforme trabalhos realizados por Mullahey et al. (1993) e Akanda et al. (1997).

As curvas-respostas para o controle do

Tabela 1 - Níveis de toxicidade na grama-batatais aos $10 \mathrm{e}$ 20 dias após o tratamento

\begin{tabular}{|l|c|c|}
\hline \multirow{2}{*}{ Tratamento } & \multicolumn{2}{|c|}{$\begin{array}{c}\text { Nível de fitotoxicidade } \\
\text { (\%) }\end{array}$} \\
\cline { 2 - 3 } & 10 DAT & 20 DAT \\
\hline Triclopyr* $\left(0,00 \mathrm{kgha}^{-1}\right)$ & 0 & 0 \\
\hline Triclopyr $\left(0,25 \mathrm{~kg} \mathrm{ha}^{-1}\right)$ & 0 & 0 \\
\hline Triclopyr $\left(0,48 \mathrm{~kg} \mathrm{ha}^{-1}\right)$ & 0 & 0 \\
\hline Triclopyr $\left(0,72 \mathrm{~kg} \mathrm{ha}^{-1}\right)$ & 0 & 0 \\
\hline Triclopyr $\left(0,96 \mathrm{~kg} \mathrm{ha}^{-1}\right)$ & 4 & 0 \\
\hline Triclopyr $\left(1,20 \mathrm{~kg} \mathrm{ha}^{-1}\right)$ & 10 & 0 \\
\hline$(2,4-\mathrm{D}+\text { picloram })^{* *}\left(480+128 \mathrm{~g} \mathrm{ha}^{-1}\right)$ & 9 & 0 \\
\hline
\end{tabular}

* Produto comercial: Garlon na concentração de $0,48 \mathrm{~g} \mathrm{~L}^{-1}$ de triclopyr.

** Produto comercial: Tordon na concentração de $240 \mathrm{~g} \mathrm{~L}^{-1}$ de $2,4-\mathrm{D}+64 \mathrm{~g} \mathrm{~L}^{-1}$ de picloram.

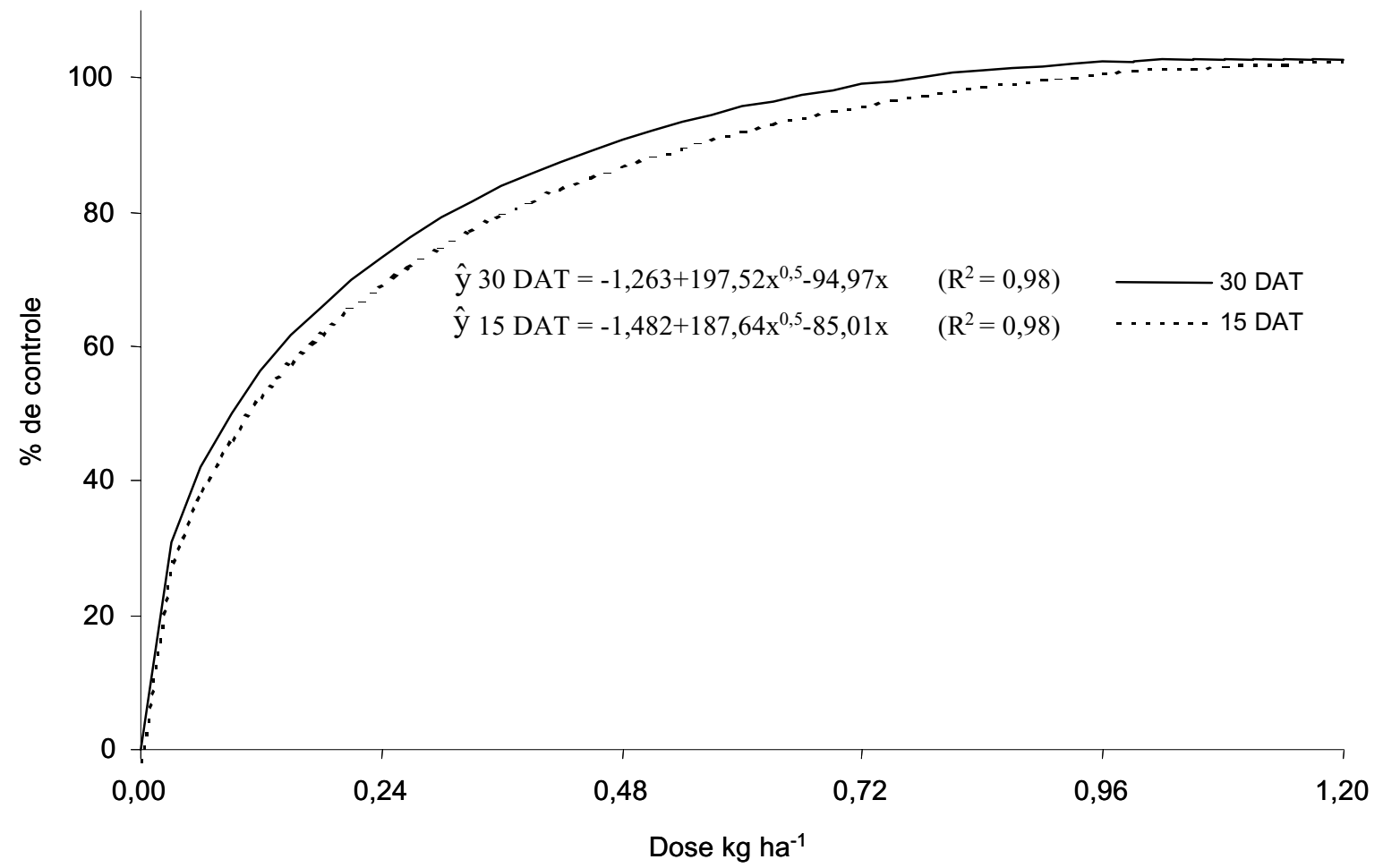

Figura 1 - Porcentagem de controle do Desmodium incanum em função da dose de triclopyr, para as avaliações aos 15 e 30 dias após o tratamento. 
Desmodium incanum, aos 15 e 30 DAT, em função da dose do triclopyr, com as respectivas equações e R2, estão apresentadas na Figura 1. O nível de controle satisfatório (90\%) aos 30 DAT, estimado segundo o modelo estatístico, foi obtido a partir da dose de $0,48 \mathrm{~kg} \mathrm{ha}$.

A Figura 2 mostra o controle da Zornia latifolia em função das doses do triclopyr para as avaliações aos 15 e 30 DAT. Verifica-se tolerância mais acentuada ao triclopyr, uma vez que o controle satisfatório (90\%), estimado pelo modelo estatístico, foi obtido a partir de $0,66 \mathrm{~kg} \mathrm{ha}$. Constatou-se também, pelo maior distanciamento das curvas de 15 e 30 DAT, na Figura 1, efeito mais lento do herbicida, necessitando-se de mais tempo para ocorrer a morte das plantas de Zornia latifolia em relação a Desmodium incanum.

Nenhuma referência foi encontrada na literatura sobre o controle de Desmodium incanum e de Zornia latifolia com o triclopyr. No entanto, bons resultados foram obtidos a $0,60 \mathrm{~kg}$ hal para a espécie Solanum viarum (Mislevy et al., 1997) e a $0,56 \mathrm{~kg}$ hal para Robus argutus (McCartye et al., 1996).

A Figura 3 contrasta a testemunha sem controle com o tratamento triclopyr $0,72 \mathrm{~kg} \mathrm{ha}{ }^{1}$ aos 30 DAT. Verifica-se, na testemunha (Figura 3A), o gramado com alto nível de infestação de Desmodium incanum e Zornia latifolia, e, para o tratamento triclopyr a $0,72 \mathrm{~kg} \mathrm{ha}^{1}$ (Figura 3B), ausência de fitotoxicidade e eficiência no controle das plantas daninhas.

Concluiu-se que o triclopyr é um herbicida com potencial para controle de plantas daninhas dicotiledôneas em gramados constituídos pela grama-batatais, uma vez que nas doses de até $1,20 \mathrm{~kg} \mathrm{ha}^{1}$ não se verificaram danos visuais na qualidade do gramado, e, a partir de 0,48 e 0,66 kg ha ${ }^{1}$, proporcionou controles iguais ou superiores a $90 \%$ de Desmodium incanum e Zornia latifolia, respectivamente.

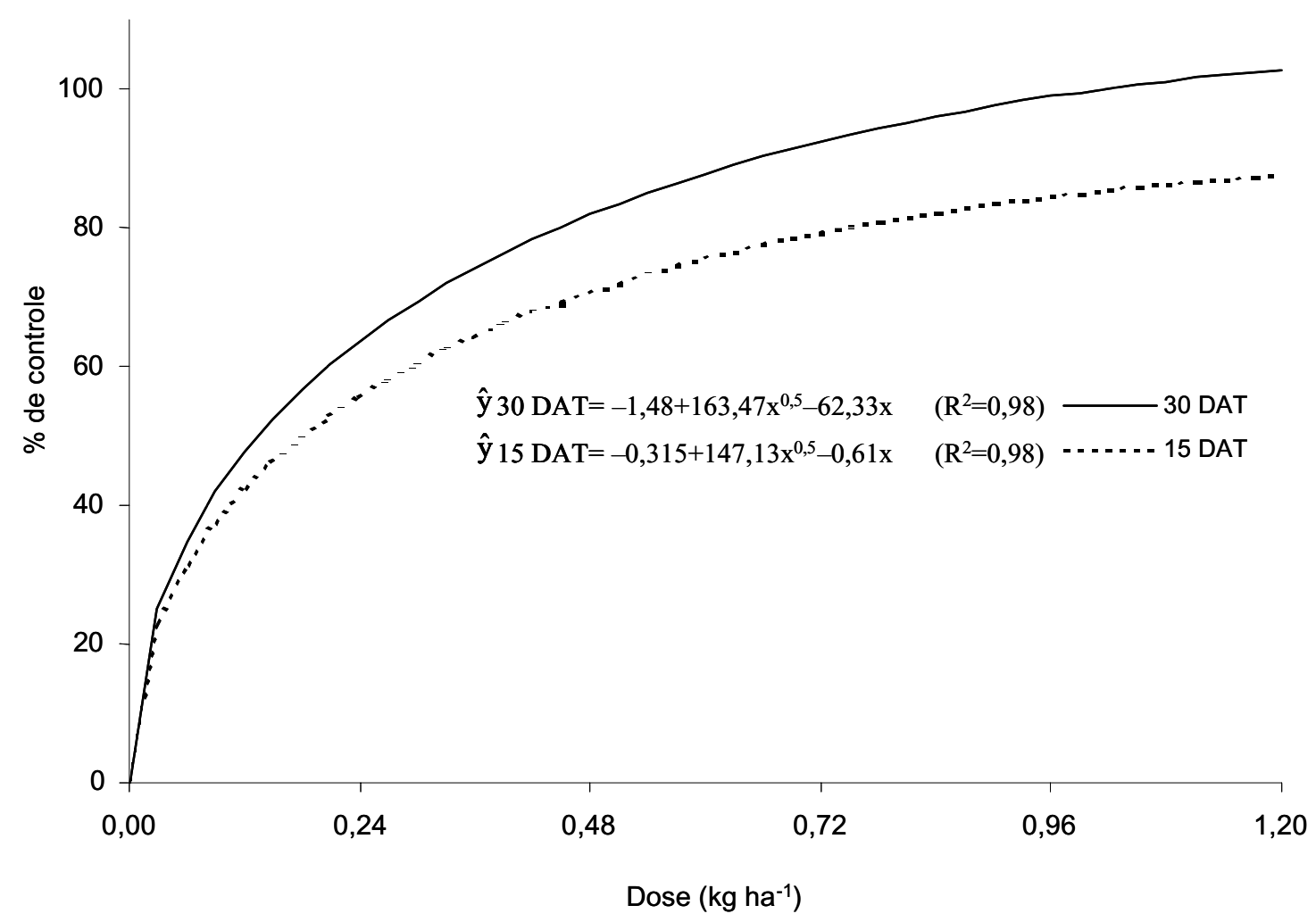

Figura 2 - Porcentagem de controle da Zornia latifolia em função da dose de triclopyr, para as avaliações de 15 e 30 dias após o tratamento. 

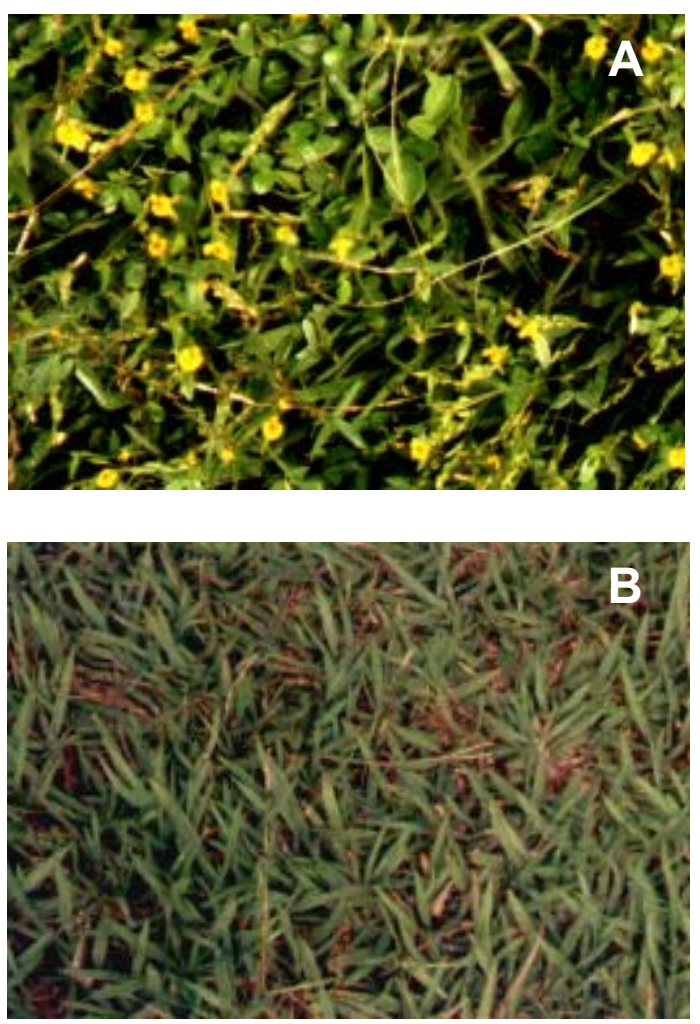

Figura 3 - Testemunha sem controle (A) e triclopyr a $0,72 \mathrm{~kg} \mathrm{ha}^{-1}(\mathrm{~B})$ aos 30 dias após o tratamento.

\section{LITERATURA CITADA}

AKANDA R. U. et al. Influence of postemergence herbicides on tropical soda apple (Solanum viarum) and Bahiagrass (Paspalum notatum). Weed Technol., v. 11, n. 4, p. 656-661, 1997.
CHRISTOFFOLETI, P. J.; ARANDA, A. N. Seletividade de herbicidas a cinco tipos de gramas. Plantas Daninhas, v. 19, n. 2, p. 273-278, 2001.

DEMATTÊ, M. E. S. P. Aplicação de nitrogênio, fósforo, potássio, adubo orgânico e calcário dolomítico na produção de sementes de grama-batatais (Paspalum notatum Flugge) em Latossol Vermelho Escuro. 1983. 34 f. Tese (Doutorado em Agronomia) - Escola Superior de Agricultura "Luiz de Queiróz”, Piracicaba, 1983.

KISSMANN, K. G. Plantas infestantes e nocivas. 2.ed. São Paulo: BASF Brasileira, 1997. p. 679-684.

LORENZI, H.; SOUZA, H. M. Plantas Daninhas do Brasil: terrestres, aquáticas, parasitas e tóxicas. Nova Odessa: Plantarum, 2000. 349 p.

McCARTYE, L. B.; COLVIN, D. L.; HIGGINS, J. M. Highbush blackberry (Robus argutus) control in and Bahiagrass (Paspalum notatum). Weed Technol., v. 10, n. 4, p. 754-761.

MISLEVY, P.; MULLAHEY J. J.; MARTIN F. G. Response of tropical soda apple (Solanum viarum) to triclopyr. Soil Crop Sci. Soc. Florida Proc., n. 56, p. 11-13, 1997.

MULlAHEY J. J.; CORNELL, J. A.; COLVIN, D. L. Tropical soda apple (Solanum viarum) control. Weed Technol., v. 71, n. 3, p. 723-727, 1993.

RODRIGUES, B. N.; ALMEIDA, F. S. Guia de herbicidas. Londrina: Edição dos Autores, 1998. 675 p.

SILVA, A. A. et al. Controle de plantas daninhas. In: ASSOCIAÇÃO BRASILEIRA DE ENSINO SUPERIORABEAS. Curso de proteção de plantas. Brasília: 2001. 260 p. (Módulo 3 ABEAS). 